\title{
An essential function for the centrosomal protein NEDD1 in zebrafish development
}

\author{
JA Manning ${ }^{1,2}$, M Lewis $^{3}$, SA Koblar ${ }^{3}$ and S Kumar, ${ }^{*, 1,2}$
}

\begin{abstract}
The centrosome is the primary microtubule organising centre of the cell. It is composed of many proteins, some of which make up the core of the centrosome, whereas others are used for specific functions. Although the cellular roles of many centrosomal proteins are well defined, much less is known about their functions and the role of the centrosome in development. In this study we investigated the function of NEDD1, a critical component of the centrosome essential for microtubule nucleation, in zebrafish (Danio rerio) development. The zebrafish homologue of NEDD1 (zNEDD1) was cloned and found to have a similar localisation and function to mammalian NEDD1. We show that zNEDD1 is essential for survival, as a high level of knockdown was embryonic lethal. Partial knockdown of ZNEDD1 caused abnormalities including an increase in mitotic and apoptotic cells. Pronounced phenotypic defects were seen in the brain, with a lack of defined brain structures, incomplete neural tube formation and a disorganisation of neurons. In addition, we show that a reduction in zNEDD1 resulted in the loss of $\gamma$-tubulin at the centrosome. Our data thus demonstrate that ZNEDD1 is critical for the recruitment of $\gamma$-tubulin to the centrosome, and is essential for the proper development of zebrafish.
\end{abstract}

Cell Death and Differentiation (2010) 17, 1302-1314; doi:10.1038/cdd.2010.12; published online 12 February 2010

The centrosome is the major microtubule organising centre (MTOC) of cells and serves as a centralised location for controlling many cellular processes. The centrosomal protein neural precursor cell expressed developmentally downregulated gene 1 (NEDD1), ${ }^{1}$ functions in the centrosomal and mitotic-spindle recruitment of the $\gamma$-tubulin ring complex $(\gamma$-TuRC). This complex is required for the nucleation of microtubules, correct formation of the mitotic spindle and hence progression of the cell cycle. ${ }^{2,3}$ Because of this, depletion of NEDD1 from mammalian cells results in dispersion of the MTOC, impaired centrosomal and chromatin nucleation of microtubules, mitotic chaos and cell-cycle arrest. $^{2-4}$

Given the centrosomal localisation and function of NEDD1, and its dynamic expression during mouse embryogenesis, ${ }^{5}$ it is expected that this protein has an important role during development. However, because of the early embryonic lethality of mouse knockouts of centrosomal proteins, including $\gamma$-tubulin, it has not been possible to study the function of these proteins in later stages of development in this organism. $^{6,7}$ Zebrafish (Danio rerio) posses many benefits for developmental studies including the conservation of genes involved in cell growth and proliferation, ${ }^{8}$ the rapid maturation of translucent embryos ${ }^{9}$ and the ability to specifically deplete proteins of interest. ${ }^{10}$ Although there has been much work analysing the cell cycle in zebrafish, ${ }^{11}$ there has been limited analysis of the centrosome in this species. Therefore, this system was chosen as a model to study the role of NEDD1 during development. In addition, NEDD1 homologues have been described in Drosophila, Xenopus and Arabidopsis. ${ }^{12-14}$ Despite the consistent centrosomal localisation of NEDD1 in these species, its effect on $\gamma$-tubulin recruitment appears to be species dependant. Hence, this study also aimed to compare the function of zebrafish NEDD1 (zNEDD1) to other homologues.

In this paper we report on the characterisation of zNEDD1 and provide evidence that this protein displays a similar localisation and function to mammalian NEDD1. In addition, we demonstrate that zNEDD1 is essential in zebrafish and its depletion leads to defects in mitosis, apoptosis and development, focusing on the brain of NEDD1 morphant embryos.

\section{Results}

Cloning of zNEDD1. A zebrafish homologue of NEDD1 was identified by a BLAST search using the human NEDD1 protein sequence. This revealed a previously uncharacterised zebrafish protein of 676 amino acids (accession number: NP_998671), that is 44\% identical and $60 \%$ similar to human NEDD1 (Supplementary Figure S1a). The 79 residues at the $\mathrm{C}$-terminus of these proteins are $68 \%$ identical and $99 \%$ similar, suggesting functional conservation

\footnotetext{
${ }^{1}$ Department of Haematology, Centre for Cancer Biology, SA Pathology, Frome Rd, Adelaide, SA 5000, Australia; ${ }^{2}$ Department of Medicine, University of Adelaide, Adelaide, SA 5005, Australia and ${ }^{3}$ Centre for the Molecular Genetics of Development, Schools of Medicine and Molecular Biomedical Science, University of Adelaide, Adelaide, SA 5005, Australia

${ }^{*}$ Corresponding author: S Kumar, Department of Haematology, Centre for Cancer Biology, SA Pathology, PO Box 14, Rundle Mall, Adelaide, SA 5000, Australia. Tel: + 6188222 3738; Fax: + 6188222 3162; E-mail: sharad.kumar@ health.sa.gov.au

Keywords: NEDD1; centrosome; zebrafish; development

Abbreviations: $\gamma$-TuRC, $\gamma$-tubulin ring complex; AP, alkaline phosphatase; DIC, differential interference contrast; DIG, digoxigenin; DTT, 1, 4-dithiothreitol; EDTA, ethylenediaminetetra-acetic acid; GST, glutathione-S-transferase; hpf, hours post fertilisation; HRP, horseradish peroxidase; MO, morpholino; MTOC, microtubule organising centre; ORF, open reading frame; PBS, phosphate-buffered saline; PBST, PBS-Tween20; PFA, paraformaldehyde; pH3, phosphorylated histone H3; PVDF, polyvinylidene difluoride; RB, Rohon-Beard; RT, room temperature; SDS, sodium dodecyl sulphate; SDS-PAGE, SDS-polyacrylamide gel electrophoresis Received 24.8.09; revised 18.12.09; accepted 12.1.10; Edited by E Baehrecke; published online 12.2.10
} 
and further indicating that this protein is the zebrafish homologue of NEDD1. As in human NEDD1, which has seven WD40 motifs in the N-terminal half of the protein, zNEDD1 also contains seven WD40 motifs lying within a similar region of the protein (Supplementary Figure S1b). These motifs are normally involved in protein-protein interactions. ${ }^{15} \mathrm{~A}$ phylogenetic analysis of this protein with known NEDD1 homologues from other species shows that Danio rerio NEDD1 shares a common ancestor with multiple species, and is closest to Xenopus laevis in evolutionary distance (Supplementary Figure S1c).

zNEDD1 expression is high in the nervous system and downregulated in development. In situ hybridisation analysis showed that in early embryogenesis zNEDD1 mRNA was distributed uniformly throughout all developing tissues (Figure 1a). This expression was increased during the $75 \%$ epiboly and bud stages of the embryo, before

a

anti-sense $Z N E D D 1$

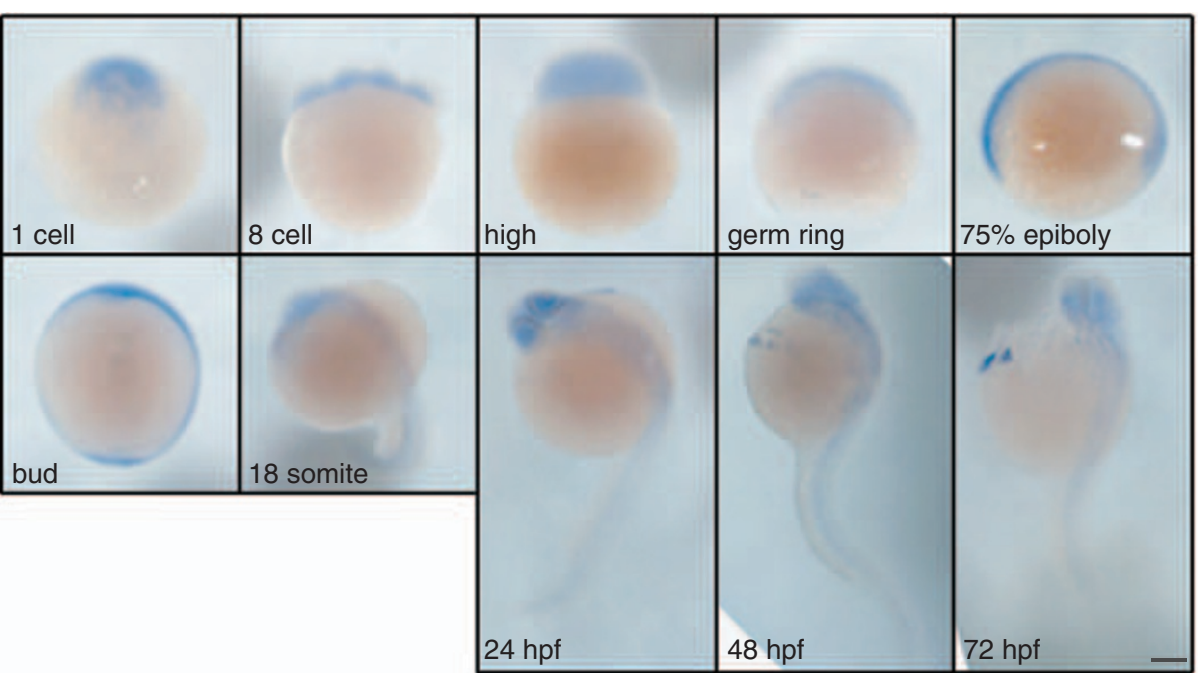

sense $z N E D D 1$

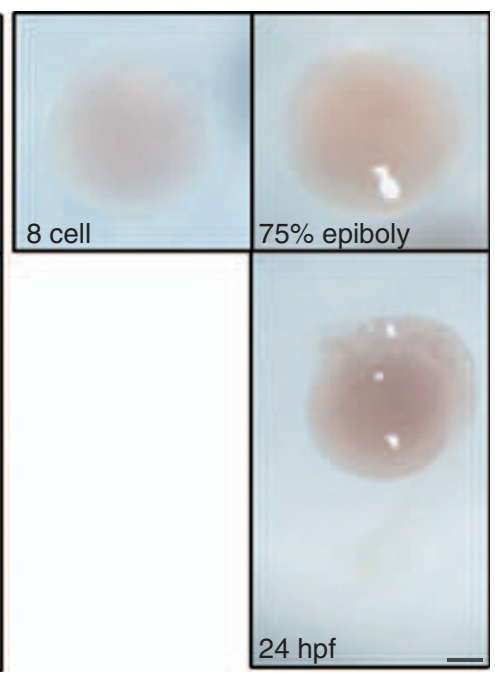

b anti-sense $z N E D D 1$

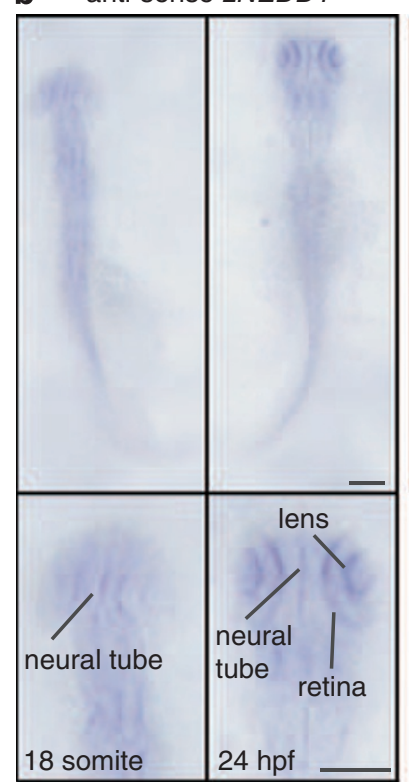

C

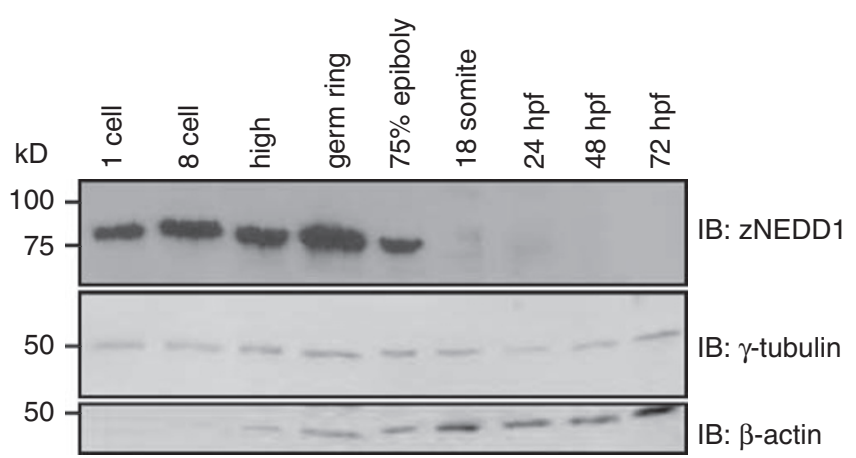

Figure 1 Zebrafish NEDD1 (zNEDD1) expression during development. Expression of ZNEDD1 mRNA was detected by in situ hybridisation using anti-sense or sense probes (a and $\mathbf{b}$ ). Scale bars $=100 \mu \mathrm{m}$. (a) In early developmental stages (one cell to germ ring) $z N E D D 1$ is ubiquitously expressed. This expression increases in cells at the $75 \%$ epiboly and bud stages, and decreases as development proceeds. No staining is present in embryos probed with a sense control (eight cells, $75 \%$ epiboly and 24 hours post fertilisation (hpf) representative stages shown). (b) After dissection of the yolk sac, dorsal views of embryos show that zNEDD1 expression is concentrated along the neural tube at the 18 somite stage and also in the polarised cells of the lens and retina at the $24 \mathrm{hpf}$ stage. This is more evident at higher magnification (right panels). No staining is present in the sense controls at the 18 somite and $24 \mathrm{hpf}$ stages. (c) Zebrafish embryos were collected at various hpf and protein extracted for immunoblot analysis after removal of the yolk. There is a high level of zNEDD1 maternal protein (first three stages), and also at the beginning of zygotic translation (germ ring). This is downregulated from the $75 \%$ epiboly stage, and is barely detectable from the 18 somite stage. Levels of $\gamma$-tubulin remain constant. Levels of $\beta$-actin remain consistent after expression becomes detectable 
a

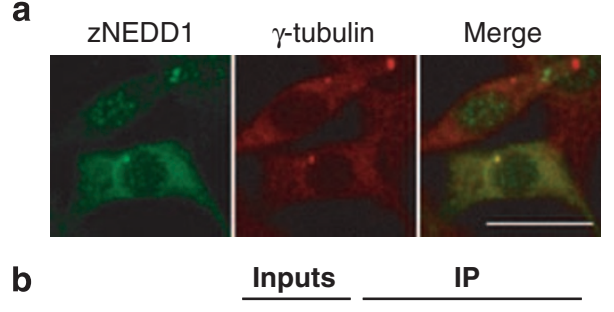

b

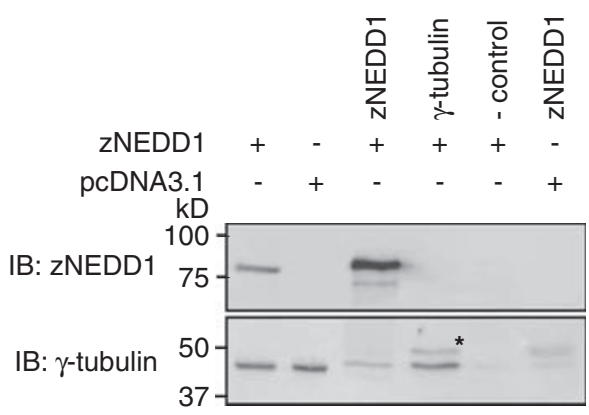

C
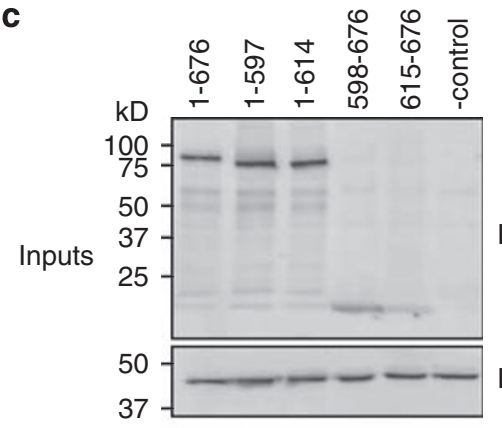

IB: Myc

IB: $\gamma$-tubulin

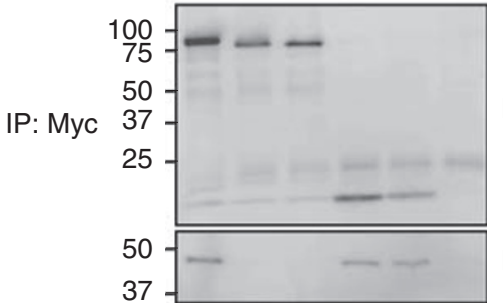

IB: Myc

IB: $\gamma$-tubulin

Figure 2 Zebrafish NEDD1 (zNEDD1) localises to the centrosome and interacts with $\gamma$-tubulin. (a) pcDNA3.1-zNEDD1 was transfected into NIH-3T3 cells and zNEDD1 localisation assessed by immunostaining. In transfected cells (lower cell only), zNEDD1 colocalises with $\gamma$-tubulin at the centrosome, with a small amount detectable in the cytoplasm. Scale bar $=40 \mu \mathrm{m}$. (b) pcDNA3.1-zNEDD1 or pcDNA3.1 alone was transfected into HEK293T cells and assessed for an interaction with endogenous $\gamma$-tubulin. Expression of zNEDD1 and $\gamma$-tubulin is confirmed in the input lysates (one-twentieth lysates loaded). The zNEDD1 antibody efficiently immunoprecipitates zNEDD1. $\gamma$-tubulin is also immunoprecipitated with ZNEDD1 and with the $\gamma$-tubulin antibody. In the absence of antibody or in cells transfected with pcDNA3.1 alone, no $\gamma$-tubulin is immunoprecipitated. The $\sim 50 \mathrm{kDa}$ band $\left(^{*}\right)$ represents $\mathrm{lgG}$. (c) The interaction of truncated forms of ZNEDD1 and $\gamma$-tubulin was assessed using endogenous $\gamma$-tubulin and Myc-tagged zNEDD1 constructs. The expression of all zNEDD1 constructs and $\gamma$-tubulin is confirmed in the inputs (one-twentieth lysates loaded). $\gamma$-tubulin was coprecipitated with full length (1-676 aa), 598-676 aa and 615-676 aa zNEDD1 only. No construct was transfected in the control lane

decreasing again as the embryo progressed through development. By $72 \mathrm{~h}$ post fertilisation (hpf) there was a marked reduction in $z N E D D 1$ expression. Upon analysis of higher magnification dorsal views of embryos at the 18 somite and $24 \mathrm{hpf}$ stages, it was apparent that $z N E D D 1$ expression was highest in neural tissues (Figure 1b). This was most evident in the neural tube at the 18 somite stage, and also in the retina and the lens of the eye at $24 \mathrm{hfp}$. This widespread expression within the developing nervous system correlates with the highest concentration of proliferating cells within the embryos at these developmental stages. ${ }^{16}$ In order to analyse zNEDD1 protein levels, an antibody against the C-terminal 252 amino acids of zNEDD1 was generated (Supplementary Figure S2). This antibody was not suitable for whole-mount staining, and hence the developmental expression of ZNEDD1 protein was assessed by immunoblotting. There was a high level of maternal zNEDD1 evident until the midblastula transition (Figure 1c, first three lanes). ${ }^{17,18}$ Consistent with the in situ data, the zygotic level of ZNEDD1 remained high early in development and was then downregulated from about the $75 \%$ epiboly stage (Figure 1c and Supplementary Figure S3). From the 18 somite stage it was barely detectable by immunoblotting, which is consistent with restricted in situ expression. As expected, $\gamma$-tubulin levels remained constant during the time course, given that it has shown to be expressed at similar levels during all the stages of development. ${ }^{19}$ As a loading control, $\beta$-actin maternal expression was low, but was upregulated from the time of zygotic expression (high stage) and remained fairly constant during development.
ZNEDD1 localises to the centrosome and interacts with $\gamma$-tubulin. When transfected into mammalian cells, zNEDD1 localised primarily to the centrosomes (Figure 2a, lower cell transfected only), where there was complete colocalisation with $\gamma$-tubulin. A small amount was also present in the cytoplasm, which is presumably due to overexpression of the protein. To examine the interaction of ZNEDD1 with $\gamma$-tubulin, zNEDD1 was immunoprecipitated from transfected cells and blotted for $\gamma$-tubulin. A clear interaction between zNEDD1 and $\gamma$-tubulin was evident (Figure 2b). An alignment of the mammalian and zebrafish proteins reveals that the Cterminal region (residues 572-660) of human NEDD1 initially found to interact with $\gamma$-tubulin, ${ }^{3}$ shows a high degree of homology with residues 598-676 of zNEDD1 (Supplementary Figure S1a). In addition, a smaller region of zNEDD1 comprising $615-676$ aa is $70 \%$ identical and $98 \%$ similar to human NEDD1. Upon immunoprecipitation of zNEDD1, $\gamma$-tubulin was found to interact with full-length zNEDD1 (1-676 aa), and constructs containing amino acids 598-676 or 615-676 (Figure 2c). No $\gamma$-tubulin was immunoprecipitated by other constructs lacking the Cterminus of zNEDD1 (residues 1-597 and 1-614). Thus, residues $615-676$ of ZNEDD1 are sufficient for its interaction with $\gamma$-tubulin.

zNEDD1 depletion in embryos leads to deformed embryos and brain disorganisation. To determine the function of zNEDD1 during development, the effect of reducing ZNEDD1 protein in early embryogenesis was examined. Two morpholinos (MOs) were designed to block the translation of zNEDD1 (Supplementary Figure S4a). 
Embryos injected with a high dose $(1 \mathrm{mM})$ of zNEDD1 MOs did not survive to $24 \mathrm{hpf}$, so lower doses of $\mathrm{MO}$ were injected. When $0.25 \mathrm{mM}$ of $\mathrm{MO} 1$ or $\mathrm{MO} 2$ alone was injected into embryos, there was $\sim 50 \%$ reduction in zNEDD1 protein levels (Supplementary Figure S4b and c). When the two MOs were coinjected $(0.125 \mathrm{mM}$ each), there was $\sim 80 \%$ depletion of zNEDD1 protein. The levels of both $\gamma$-tubulin and $\beta$-actin were unchanged.

When compared with control-injected embryos, those injected with an intermediate dose of zNEDD1 $\mathrm{MO} 1+2$ ( $0.25 \mathrm{mM}$ total) mostly survived to $24 \mathrm{hpf}$, but appeared unhealthy with an apparent accumulation of cell death (Figure 3). In the brain, there was obvious disorganisation of normal patterning and size such that the overall structure of the heads was smaller. Hence, this concentration of MO was chosen for further analysis. At $24 \mathrm{hpf}$, the uninjected and control-injected embryos developed normally and many clearly defined structures of the brain such as the ventricles (tectal and fourth ventricle labelled) and the cerebellum could be observed (Figure $3 a$ and $b$ ). The forebrain, the midbrain and the hindbrain could also be identified and their boundaries easily distinguished. In addition, the eyes were easily delineated. All zNEDD1-depleted embryos displayed abnormalities, but there was some variability in the extent of these defects, presumably depending on the efficiency of knockdown (Figure $3 \mathrm{c}-\mathrm{e}$ ). It was possible to arbitrarily categorise the zNEDD1 morphants into mild, moderate and severely abnormal phenotypes, with the majority of zNEDD1-depleted fish displaying moderate levels of abnormalities. When compared with uninjected and control injected, all zNEDD1depleted embryos displayed abnormal shortened tails with a curved body axis; however, defects in the nervous system were analysed further in this study (Figure 3c-e). In cases of mild abnormalities, the eyes remained largely intact, and the midbrain and the hindbrain were present and well defined (Figure 3c). However, the forebrain was not properly developed. This phenotype was more obvious in cases of moderate abnormalities, with the forebrain appearing severely disorganised and containing aggregates of dark masses, presumably representing dead cells (Figure $3 d$ ). The ventricular system was also abnormal in size and shape, although the midbrain and the hindbrain structures were still defined, and eyes were present. In zNEDD1-depleted embryos classed as being severely abnormal, the eyes were completely obliterated (Figure 3e). There were more dark masses, and the midbrain, the hindbrain and the forebrain structures could not be delineated. These results indicate that the knockdown of NEDD1 leads to abnormalities including in patterning and organisation of the zebrafish brain.

Phenotypes of zNEDD1-depleted embryos can be rescued by $Z$ NEDD1 $\mathrm{mRNA}$. In order to verify that the observed phenotypes were because of specific zNEDD1 knockdown and not off-targets effects, the combined MOs were injected into embryos together with capped full-length $z N E D D 1$ mRNA. It is important to note that co-injection with zNEDD1 mRNA largely rescued the phenotypes, such that these embryos appeared similar to uninjected embryos (Figure 3f). When compared with uninjected embryos, embryos injected with zNEDD1 MO1 + 2 displayed obvious phenotypic abnormalities with only $\sim 30 \%$ appearing normal (Figure $3 \mathrm{~g}$ ). However, upon co-injection of zNEDD1 MO1+2 with zNEDD1 mRNA, $\sim 80 \%$ of embryos appeared morphologically normal. The rescue of morphology was shown to be because of the significant restoration of zNEDD1 protein levels by the co-injection (Figure 3h). These results confirm that the reduction in zNEDD1 caused by $\mathrm{MO}$ injection is specifically responsible for the observed phenotypic abnormalities.

zNEDD1-depleted embryos display a high level of apoptosis. The dark cell masses apparent in the phenotypic analysis of zNEDD1-depleted embryos suggested that these morphants displayed a high level of cell death. To investigate this further, embryos were assessed for apoptosis by TUNEL. Although uninjected and control-injected embryos exhibited very few apoptotic cells (18 \pm 4 and $18 \pm 7$ TUNEL positive cells per embryo respectively, Figure $4 a, b$ and f), zNEDD1-depleted embryos consistently displayed a higher level of apoptosis present throughout the whole embryo $(64 \pm 12$ TUNEL positive cells per embryo), but more concentrated in the brain and the neural tube (Figure $4 c^{\prime}, c^{\prime \prime}$ and f). In particular, there were a high number of apoptotic cells in the forebrain, correlating with the gross morphological abnormalities and cell death seen in this region of the brain in Figure 3. Co-injection with $z N E D D 1$ mRNA rescued this phenotype (18 \pm 7 TUNEL positive cells per embryo, Figure $4 d$ and $f$ ), thus indicating that the apoptosis is caused by the specific reduction of zNEDD1. It is known that some MOs nonspecifically induce p53 activity, which may result in increased cell death. ${ }^{20}$ In order to discount this as the cause for the apoptosis seen when injecting with zNEDD1 MOs, co-injections were carried out with a previously characterised p53 MO. ${ }^{20,21}$ After co-injection of zNEDD1 MOs with the p53 MO, apoptosis was still occurring, although the number of TUNEL-positive cells appeared to be slightly lower in the co-injected embryos (56 \pm 5 TUNEL-positive cells per embryo, Figure $4 \mathrm{e}$ and $\mathrm{f}$ and Supplementary Figure S5). As this phenotype was rescued by co-injection with ZNEDD1 mRNA, this suggests that some of the apoptosis in embryos depleted of zNEDD1 by MO injection relies on p53 activity.

zNEDD1-depleted embryos display mitotic arrest. Given that the depletion of NEDD1 in cultured mammalian cells has been shown to cause mitotic arrest, ${ }^{2,3}$ we examined whether there were any changes in the mitotic index of zNEDD1depleted zebrafish embryos by staining for phosphorylated histone $\mathrm{H} 3(\mathrm{pH} 3)$, which is present during $\mathrm{G} 2$ and mitosis. ${ }^{22}$ zNEDD1-depleted embryos displayed an increase in the number of mitotic cells when compared with uninjected or control-injected embryos (75 \pm 12 pH3-positive cells per embryo for uninjected, $71 \pm 14$ for control and $150 \pm 42$ for zNEDD1-depleted, Figure 5a-c and e). As there did not appear to be an increase in total cell numbers or size of the embryos, this suggests that many cells may have been arrested in mitosis. The mitotic cells were present throughout the embryo, but appeared concentrated in the brain and neural tube. Co-injection with $z N E D D 1$ mRNA partially 
rescued this phenotype, although unlike other phenotypes this was not completely restored, $(114 \pm 16 \mathrm{pH} 3$ positive cells per embryo, Figure $5 d$ and e). This may be explained by the incomplete restoration of zNEDD1 protein levels following zNEDD1 mRNA injection, which may have been insufficient to avert the arrest of some cells in mitosis.

a
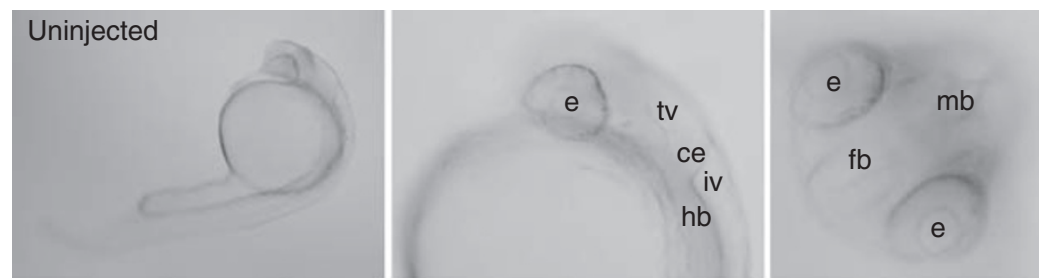

b
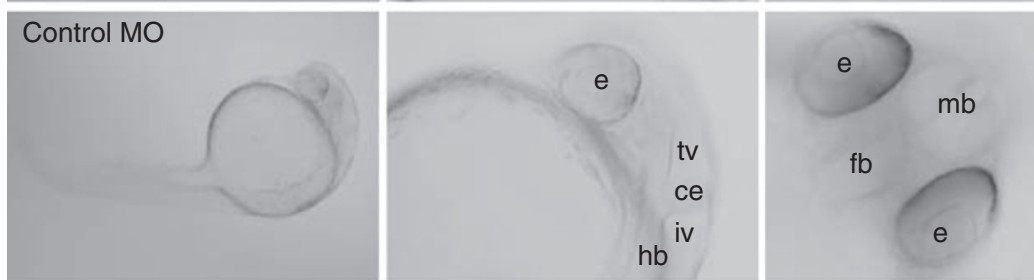

C
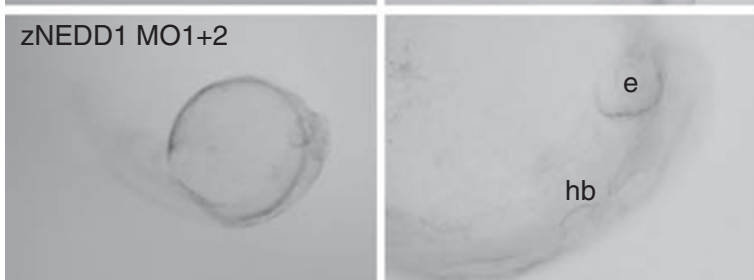

d
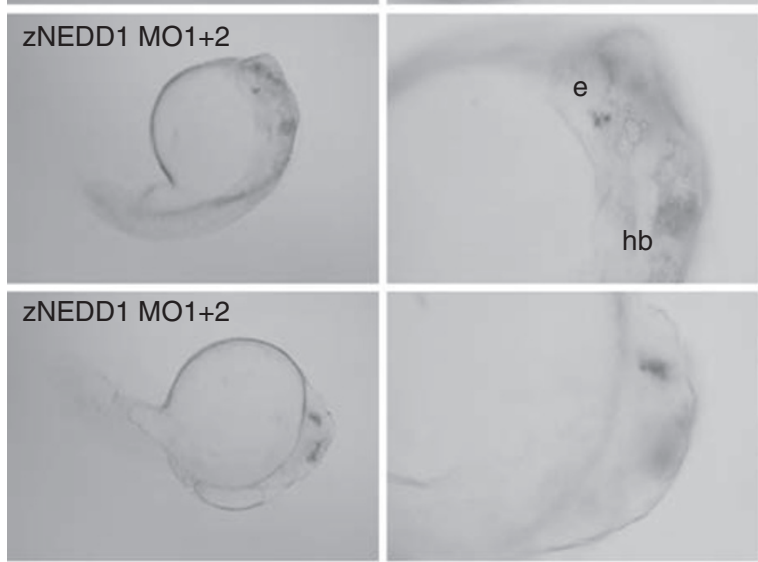

f
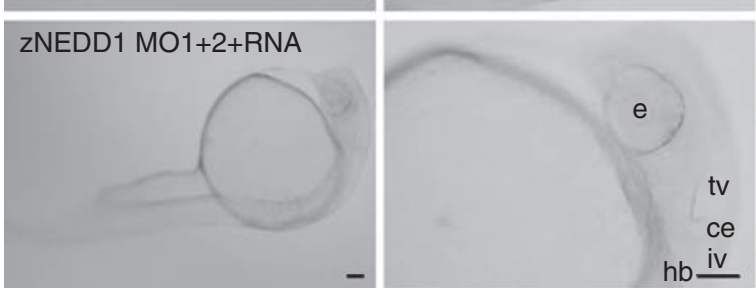

g
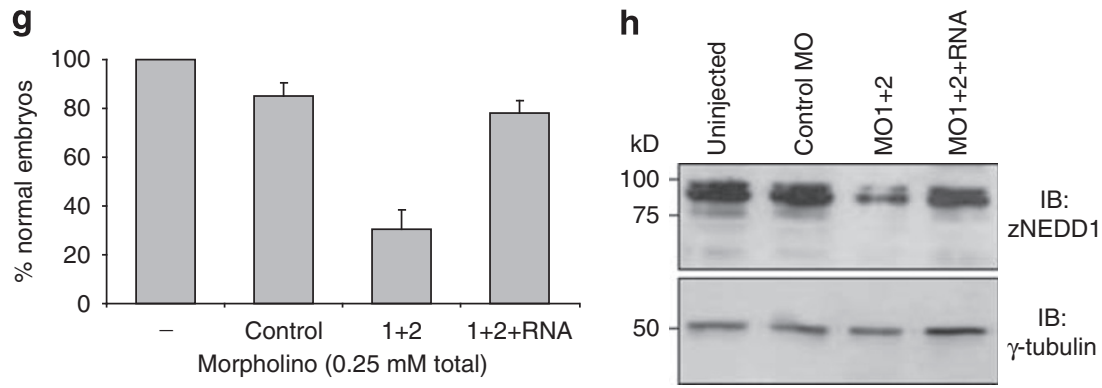
Overexpression of residues 598-676 of zNEDD1 mimics the apoptosis and mitotic arrest of zNEDD1 depletion. We have recently observed that the $\mathrm{C}$-terminal region of mammalian NEDD1 acts as a dominant-negative by binding to endogenous $\gamma$-tubulin and sequestering it away from the centrosome (JAM and SK, unpublished data). As a similar region of zNEDD1 is sufficient for binding to $\gamma$-tubulin (Figure 2c), we tested whether overexpression of residues 598-676 of zNEDD1 could cause effects similar to those seen by zNEDD1 depletion. It is interesting to note that $76 \%$ of embryos injected with GFP-tagged zNEDD1 598-676 mRNA were dead or severely abnormal by $24 \mathrm{hpf}$, compared with only $20 \%$ of embryos injected with GFP mRNA suggesting that overexpression of GFP-zNEDD1 598-676 is toxic to the embryos. However, in surviving embryos, those with detectable GFP-zNEDD1 expression, but not GFP control, were phenotypically deformed, similar to the embryos injected with zNEDD1 $\mathrm{MO}$ in Figure 3 (Supplementary Figure S6a). In addition, these embryos displayed a higher level of TUNEL and $\mathrm{pH} 3$ staining indicative of increased apoptosis and mitotic arrest, although this was not quantitated given the severity of abnormalities and low number of surviving embryos (Supplementary Figure $\mathrm{S} 6 \mathrm{~b}$ and $\mathrm{c}$ ). This suggests that residues 598-676 of zNEDD1 act as a dominant negative to disrupt normal zNEDD1 function and provides further evidence that zNEDD1 is important for zebrafish development.

zNEDD1-depleted embryos undergo neurogenesis, but present poorly patterned neuronal structures. Given the mitotic arrest and increased apoptosis in zNEDD1-depleted embryos, it was no surprise that these embryos displayed other morphological abnormalities. Also, consistent with the expression of $z N E D D 1$ mRNA in neural tissues (Figure 1b), the disorganised appearance of brains in the zNEDD1 morphants (Figure 3) was anticipated and further suggested that these embryos may exhibit neuronal defects. To characterise this further, embryos at $24 \mathrm{hpf}$ were stained with an antibody to $\mathrm{HuC}$, a protein that maintains high expression in post-mitotic cells in most regions of the nervous system. ${ }^{23}$ All embryos were developmentally age matched, as can be seen by the normal development of structures such as the eyes in most cases (Figure 6). In uninjected, control injected and zNEDD1 MO1 + 2+ RNA co-injected embryos, HuC was expressed in multiple regions of the brain and in a continuous population of post-mitotic neurons throughout the rostrocaudal axis of the neural tube (Figure $6 \mathrm{a}, \mathrm{b}$ and $\mathrm{d}$, arrows). ${ }^{24,25}$ These neurons were still present in zNEDD1-depleted embryos, even though the embryos had growth defects and abnormalities at this stage (Figure 6c). Upon closer analysis of the staining in the trunk of these embryos, the HuC-positive neurons consistently appeared similar in number and patterning to uninjected, control and rescued embryos (Figure 6e-h). However, in regions of the brain, the hindbrain and the cranial ganglia, there were variable effects of zNEDD1 depletion on neuronal number and patterning, with the majority of embryos displaying a moderate phenotype. In a dorsal view of embryos at $24 \mathrm{hpf}, \mathrm{HuC}$ is normally distributed throughout regions of the forebrain, the hindbrain and in the cranial ganglia, as seen in uninjected, control and rescued embryos (Figure 6i, j and I). After zNEDD1 depletion, embryos that displayed moderate abnormalities still contained many HuC-positive neurons, although there was distorted patterning of brain structures and cranial ganglia (Figure $6 \mathrm{k}^{\prime}$ ). In addition, the brain structures were reduced in size, which is consistent with the increase in cell death and mitotic arrest described previously in the brain (Figure 4). In embryos that displayed severe morphological defects, HuC-positive neurons were still present, but the severity of the brain, the hindbrain and the cranial ganglia disruption to size, neuronal number and patterning was more intense (Figure $6 \mathrm{k}^{\prime \prime}$ ). Hence, zNEDD1 depletion from zebrafish embryos does not appear to affect the differentiation of post-mitotic neurons, but does cause severe defects in neuronal organisation in the brain.

To further characterise the effects of ZNEDD1 depletion on neural development, embryos were also analysed for RohonBeard (RB) sensory neurons. ${ }^{26}$ In uninjected and controlinjected embryos, characteristic acetylated $\alpha$-tubulin staining revealed $\mathrm{RB}$ cells and their axonal projections along the tracts of the dorsal longitudinal fasciculus (Figure $6 \mathrm{~m}$ and $n$ ). ${ }^{27}$ In zNEDD1-depleted embryos, there was variability in the extent of abnormalities seen. Embryos with mild and moderate phenotypes displayed a relatively normal morphology of the neural tube, but had greatly reduced acetylated $\alpha$-tubulin staining, with a decrease in RB cells and a marked reduction in the fasciculation of their axons (Figure 6o'). Severely abnormal zNEDD1 morphants displayed scarce RB cells, residing in the proximal region only. Axons were disorganised and displayed markedly reduced fasciculation, probably due

Figure 3 Zebrafish NEDD1 (ZNEDD1)-depleted embryos are severely deformed and show brain disorganisation. Embryos were viewed by light microscopy at $24 \mathrm{hpf}$. First two panels are lateral view, third panel is dorsal view. The eye (e), the tectal ventricle (tv), the cerebellum (ce), the fourth ventricle (iv), the hindbrain (hb), the forebrain (fb) and the midbrain (mb). (a and $\mathbf{b}$ ) Uninjected and control-injected embryos are identical in appearance with well-defined structures in the brain. (c-e) Most zNEDD1-depleted embryos (ZNEDD1 MO1 + 2) display a deformed phenotype, which varies between embryos. (c) In cases of mild defects, the forebrain appears disorganised, but the midbrain, the hindbrain and the eyes appear to develop normally. (d) In moderate cases, the disorganisation of the forebrain is more severe, and areas of the ventricular system also appear disturbed with patches of dark cell masses. (e) In severe cases, there are more dark cell masses, no clear delineation of the forebrain, the midbrain and the hindbrain, and the eyes are obliterated. (f) In embryos co-injected with zNEDD1 mRNA (zNEDD1 MO1 + 2+RNA), the phenotypes are rescued to a large extent and the brain structures are restored. Scale bars $=100 \mu \mathrm{m}$. (g) Embryos were injected with control morpholino (MO), the combined zNEDD1 MO1 +2 or zNEDD1 MO1 +2 together with $5 \mathrm{ng} \mu \mathrm{l}^{-1}$ capped $z N E D D 1 \mathrm{mRNA}$. Only $31 \%$ of embryos injected with zNEDD1 MO1 +2 display normal morphology resembling uninjected embryos. This is rescued by the co-injection with $z N E D D 1$ mRNA which results in $78 \%$ of embryos now appearing morphologically indistinguishable from uninjected embryos (MO1 + $2+$ RNA). The errors bars represent S.E.M., $n=3$ independent experiments using approximately 50 embryos each. (h) Protein was extracted $24 \mathrm{~h}$ after injection and $20 \mu \mathrm{g}$ of each sample immunoblotted for zNEDD1 and $\gamma$-tubulin. ZNEDD1 MO injection greatly reduces zNEDD1 levels and this is restored by co-injection with zNEDD1 mRNA. $\gamma$-tubulin serves as a loading control, as this is not affected by zNEDD1 depletion 


\section{TUNEL staining}
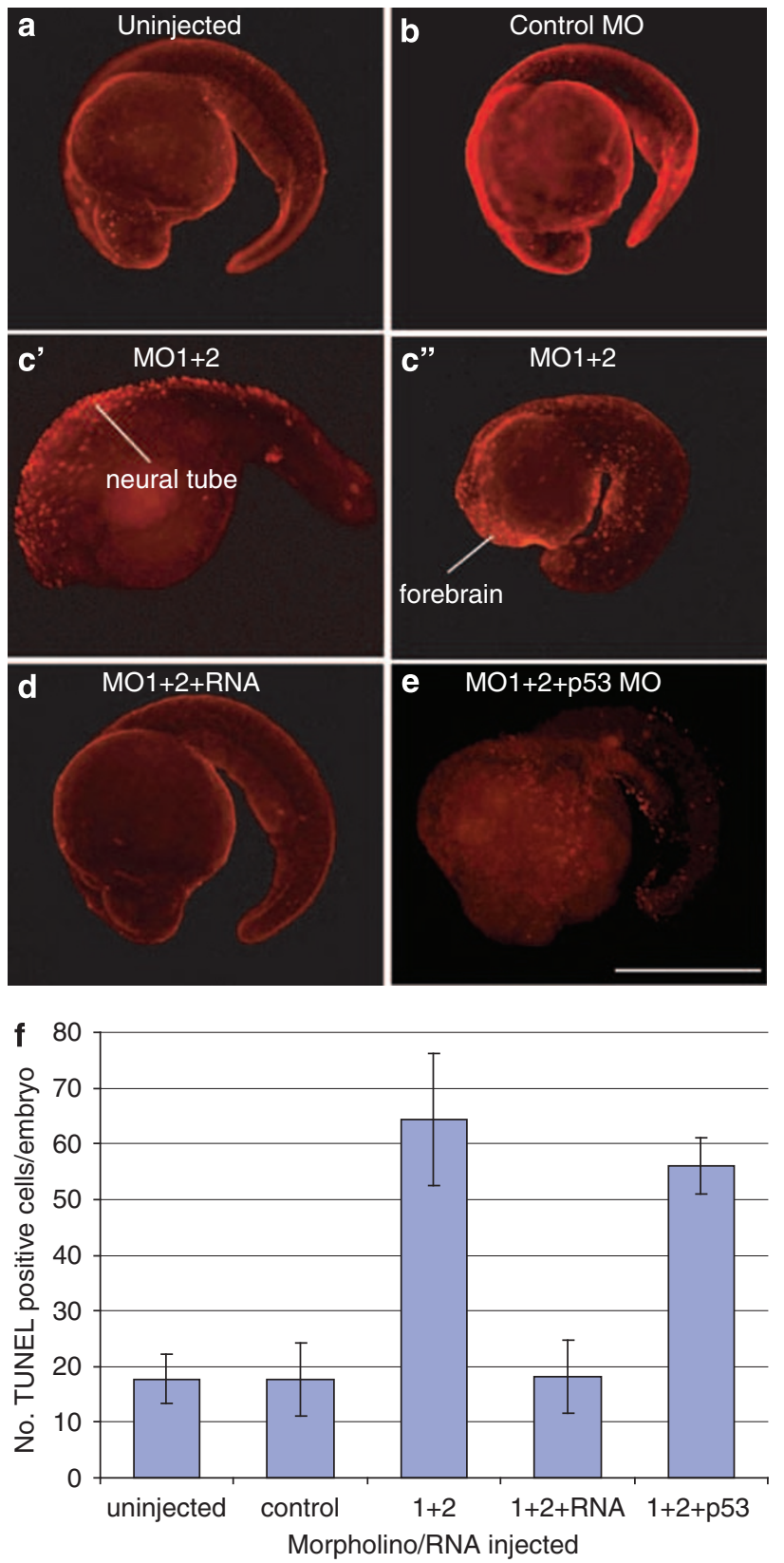

Figure 4 Zebrafish NEDD1 (zNEDD1) depletion causes a high level of apoptosis. Uninjected and morpholino (MO)-injected embryos were assessed for apoptosis by TUNEL at 24 hours post fertilisation (hpf). (a and $\mathbf{b})$ Uninjected and control injected embryos display very few TUNEL-positive cells. (c) zNEDD1 $\mathrm{MO1}+2(0.25 \mathrm{mM}$ total)-injected embryos show a high level of apoptosis, primarily concentrated in the forebrain and the neural tube. Representative examples of two embryos are shown $\left(\mathbf{c}^{\prime}\right.$ and $\left.\mathbf{c}^{\prime \prime}\right)$. (d) The apoptosis is rescued by co-injection with zNEDD1 mRNA. (e) Co-injection of ZNEDD1 MO1 + 2 and p53 MOs $(0.25 \mathrm{mM}$ $\mathrm{MO1}+2+0.5 \mathrm{mM}$ p53 $\mathrm{MO}$ ) results in apoptosis, although there are not as many TUNEL positive cells as in ZNEDD1 MO1 +2 injection alone. Scale bars $=500 \mu \mathrm{m}$. (f) Quantification of numbers of TUNEL-positive cells from five experiments, with approximately five embryos for each group. Error bars represent S.E.M. $(n=5)$

to the reduction in neurons present in these embryos (Figure $\left.60^{\prime \prime}\right)$. It was also easily observed in the acetylated $\alpha$-tubulin stained embryos that the neural tube was severely abnormal, suggesting spina bifida (incomplete closure of the neural tube) was occurring. As seen for other phenotypes, co-injection with $z N E D D 1$ mRNA rescued the defects (Figure $6 p$ ). Thus, as well as disrupting post-mitotic HuC neurons, the depletion of zNEDD1 also caused abnormalities in the number and patterning of RB sensory neurons, their axonal outgrowth and fasciculation, and neural tube formation.

Depletion of zNEDD1 causes a loss of $\gamma$-tubulin from the centrosome. In mammalian cells, the most important function of NEDD1 identified thus far is its role in recruiting $\gamma$-tubulin to the centrosome, ${ }^{2,3}$ although this does not seem to be true in Drosophila and Xenopus. ${ }^{13,28}$ It was hypothesised that the inability of $\gamma$-tubulin to localise to the centrosome in zNEDD1-depleted embryos may contribute to the phenotypic abnormalities observed in this study. Therefore, we assessed whether zNEDD1 is important in the recruitment of $\gamma$-tubulin to the centrosome in zebrafish.

At $24 \mathrm{hpf}$, in all cells of control-injected embryos, zNEDD1 and $\gamma$-tubulin perfectly co-localised in centrosomes. This was most obvious in regions of high proliferation such as in the hindbrain. The centrosomes were intensely stained with zNEDD1 and $\gamma$-tubulin at the apical surface in these cells (Figure $7 \mathrm{a}$, section through the fourth ventricle shown). In zNEDD1-depleted embryos, there was a dramatic reduction in the levels of expression of centrosomal zNEDD1 and $\gamma$-tubulin in the same region and the ventricles were less well formed (Figure 7b). Similar observations were made in the forebrain (Figure 7c and d). Thus, similar to mammalian NEDD1, zNEDD1 appears to be required for the recruitment of $\gamma$-tubulin to centrosomes. Therefore, it seems likely that the centrosomal reduction of $\gamma$-tubulin in zNEDD1-depleted embryos contributes to the observed morphological abnormalities.

\section{Discussion}

This study describes the identification and characterisation of a zebrafish homologue of NEDD1, which displays highly conserved regions of sequence similarity to mammalian NEDD1. The analysis of mRNA and protein revealed that zNEDD1 is highly expressed early in embryonic stages, particularly in neural regions, and is downregulated as embryogenesis proceeds. This observation is consistent with initial observations that NEDD1 is downregulated in the brain during mouse embryonic development. ${ }^{5}$ The high expression of $z N E D D 1$ correlates with the time of the most rapid proliferation occurring in the embryo. ${ }^{16}$ This aligns well with the expression of mammalian NEDD $1,{ }^{5}$ and suggests that zNEDD1 may be important in early development, particularly in the nervous system at times of rapid proliferation. It is important to note that zNEDD1 appears to function in a manner similar to homologues identified in mammals, ${ }^{2,3}$ Drosophila $^{12,28}$ and Xenopus ${ }^{13}$ by localising to the centrosome and interacting with $\gamma$-tubulin. However, unlike the dynamic expression of zNEDD1, the level of $\gamma$-tubulin remains constant during embryogenesis, suggesting that there may be additional $\gamma$-tubulin-independent functions of zNEDD1. It is 

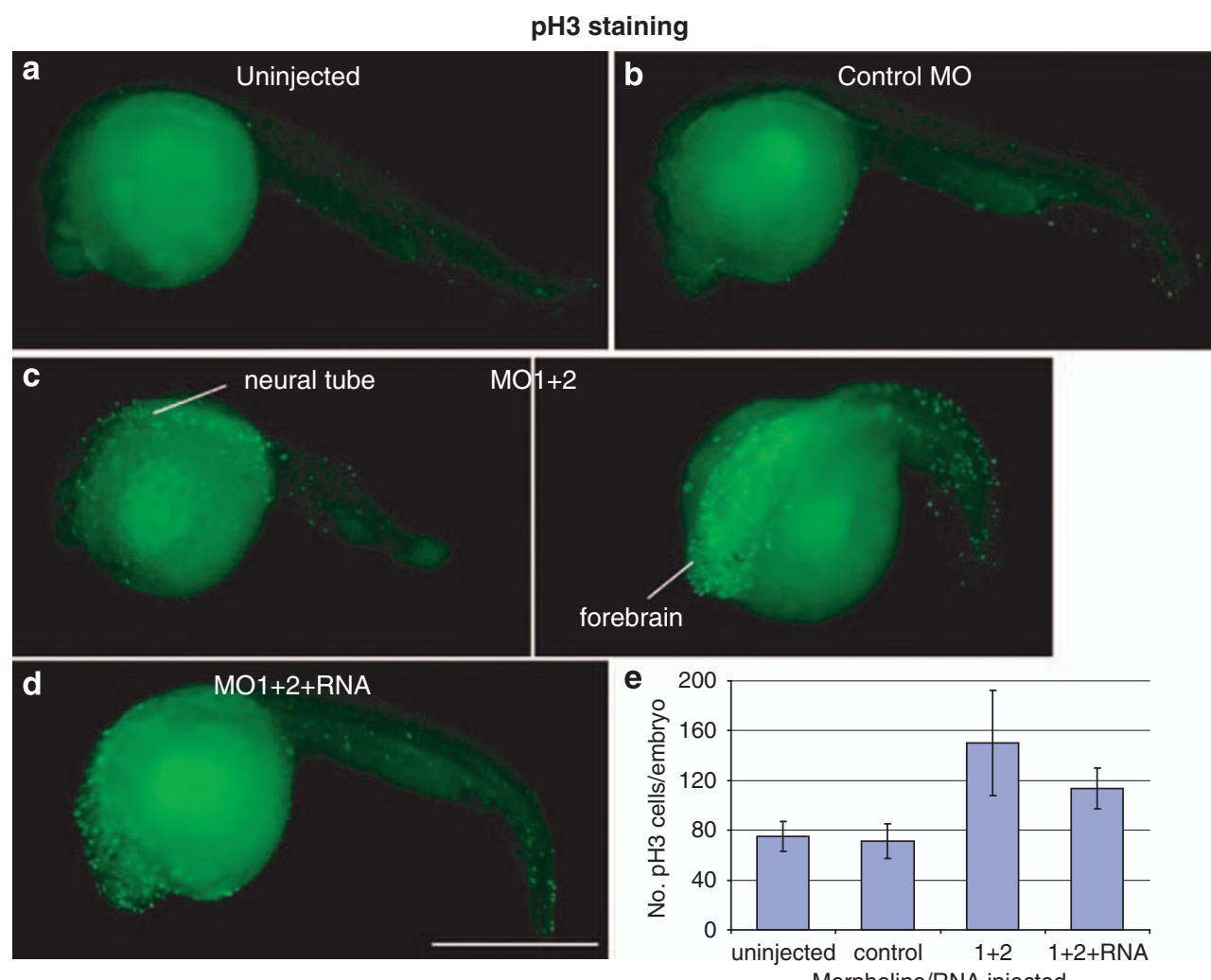

Figure 5 Zebrafish (NEDD1) (ZNEDD1) depletion causes an increase in mitotic cells. Uninjected or morpholino (MO)-injected embryos were assessed for mitotic cells by phosphorylated histone $\mathrm{H} 3(\mathrm{pH} 3)$ staining at 24 hours post fertilisation (hpf). (a and $\mathbf{b})$ Uninjected and control-injected embryos display low levels of pH3 positive cells. (c) ZNEDD1 MO1 + 2-injected embryos have an increased number of $\mathrm{pH} 3$ positive cells, primarily in the brain and the neural tube. Two representative embryos are shown. The division (white line) in the right panel indicates two different focal planes. (d) zNEDD1 MO1 + 2-injected embryos rescued with $5 \mathrm{ng} \mu \mathrm{l}^{-1}$ zNEDD1 mRNA partially restores the level of $\mathrm{pH} 3$ staining. Scale bars $=500 \mu \mathrm{m}$. (e) Quantification of numbers of pH3-positive cells with six embryos for each group. Error bars represent S.D. ( $n=6$ embryos each)

plausible that this protein is important in other cellular processes besides its established role in microtubule organisation.

Depletion of zNEDD1 resulted in embryos displaying variable phenotypes, with all exhibiting developmental and cellular abnormalities, including smaller heads, disorganised brains, evidence of spina bifida and an increase in apoptotic and mitotic cells. Defects in post-mitotic and sensory neurons were also observed, suggesting that zNEDD1 may regulate the proliferation, organisation and migration of neurons, which are processes that are known to involve the centrosome. ${ }^{29,30}$ The highest level of abnormalities was seen in the forebrain, but the reasons for this are at present unknown. It is important to note that the observed phenotypes were the result of specific ZNEDD1 depletion, as co-injection of the MOs with zNEDD1 mRNA, which restored zNEDD1 protein levels, was able to rescue the phenotypes. In addition, overexpression of a GFP-tagged C-terminal region of zNEDD1 caused similar effects as ZNEDD1 depletion although it was more toxic to the embryos. The equivalent region of mammalian NEDD1 acts as a dominant negative by binding to $\gamma$-tubulin and sequestering it away from the centrosome (JAM and SK, unpublished data). Therefore, overexpression of this region of zNEDD1 in zebrafish is likely to function by interfering with the function of endogenous zNEDD1 protein. These loss-of-function studies clearly indicate that ZNEDD1 is critical for the development and the proper formation of the brain.

Correlating with the morphological abnormalities, it was found that zNEDD1-depleted embryos displayed a high level of apoptosis. It is important to note that this occurred primarily in the brain and the neural tube, which are regions of high zNEDD1 expression. It is interesting to note that although this apoptosis still occurred after co-depletion of p53, which is often upregulated after $\mathrm{MO}$ injection, there was a slight reduction in the amount of apoptotic cells in these embryos. As many proteins induce apoptosis by stabilising or upregulating $\mathrm{p} 53,{ }^{31}$ it is feasible that zNEDD1 depletion may exert some of its effects through the p53 pathway.

In addition to cell death, consistent with the depletion of NEDD1 in mammals resulting in an increased mitotic index, ${ }^{2,3}$ zebrafish embryos with reduced zNEDD1 had a higher incidence of $\mathrm{pH} 3$ staining, indicative of mitotic arrest. Given that the increase in mitotic cells and the observed apoptosis were occurring in similar regions of the embryo, it is likely that the apoptosis was a downstream consequence of the mitotic arrest. By inhibiting cell proliferation, it has been demonstrated that the amount of proliferation occurring in the midbrain and the hindbrain neural tube surrounding the ventricles correlates with future ventricle size. ${ }^{32}$ Hence, it is plausible that the zNEDD1-depleted cells in this study may be 
arresting in mitosis and then undergoing apoptosis, therefore not allowing cell proliferation and thus resulting in smaller brain ventricular structures.

It is interesting to note that these phenotypes resemble the zebrafish cassiopeia mutant, which displays an increased mitotic index and increased numbers of apoptotic cells. ${ }^{33}$ The cassiopeia mutant harbours a loss-of-function of the SCL-interrupting locus (SIL) gene ${ }^{34}$ which is normally expressed specifically in mitosis. ${ }^{35}$ Embryos depleted of SIL have extremely disorganised mitotic spindles and often lack one
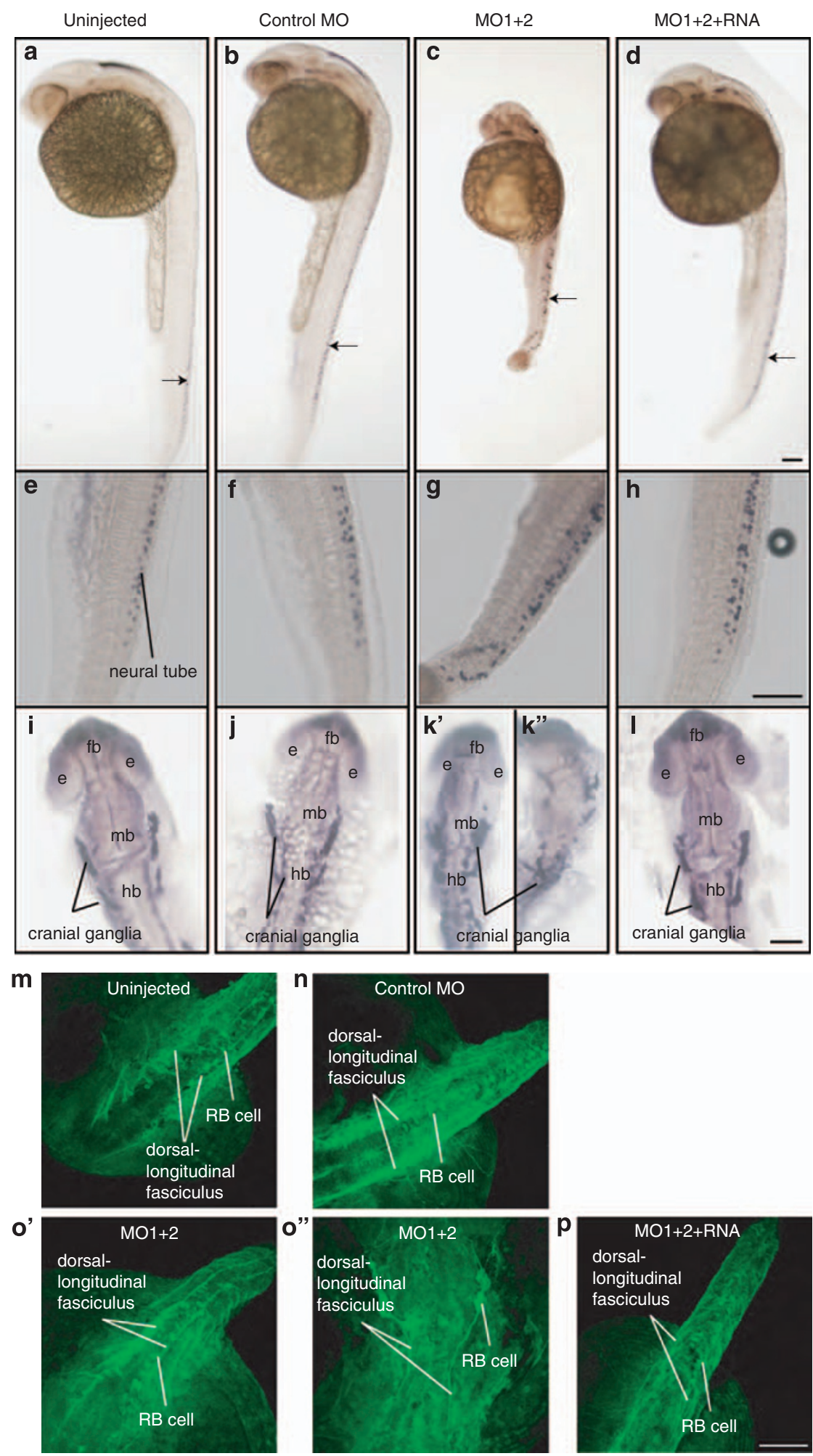
a

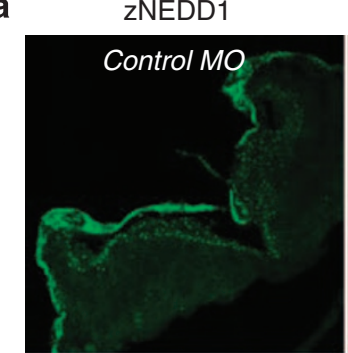

b

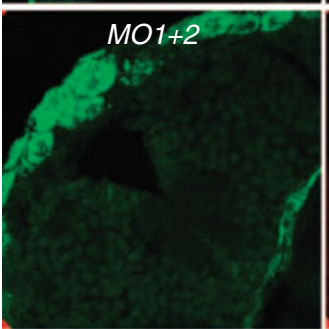

c

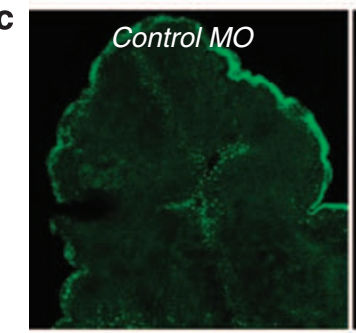

d

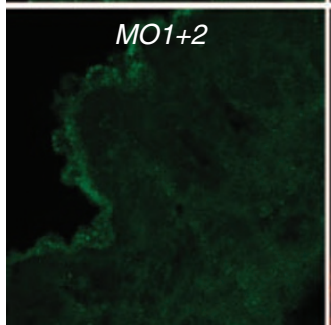

$\gamma$-tubulin
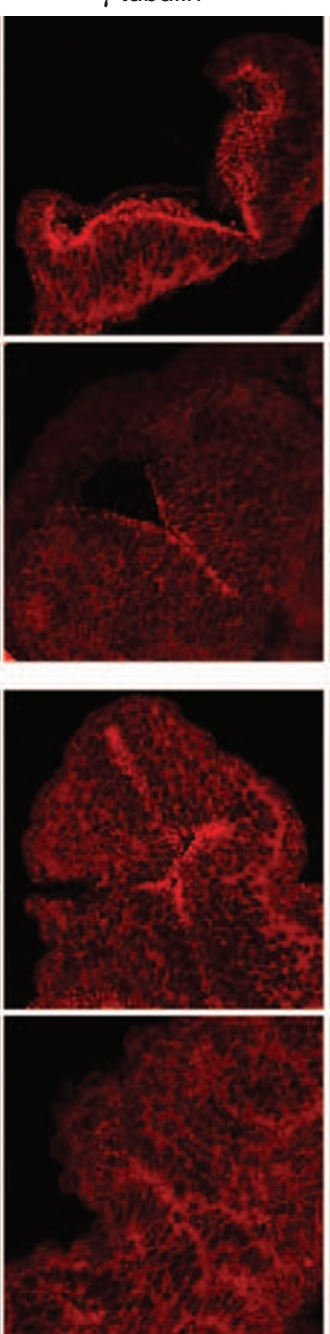
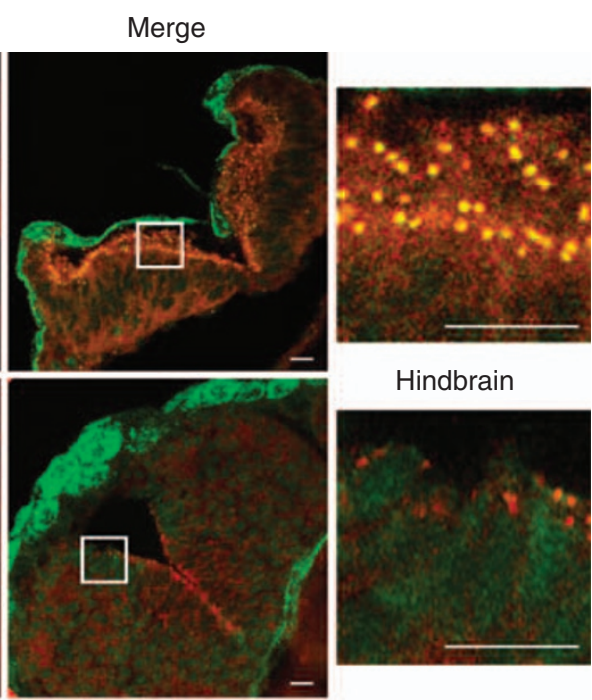

Hindbrain


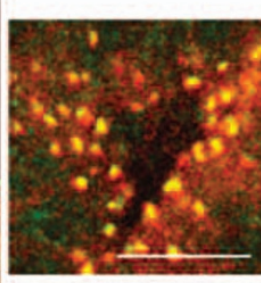

Forebrain

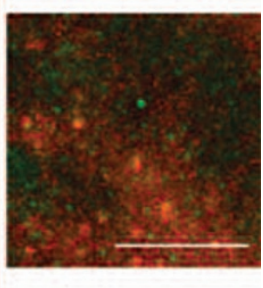

Figure 7 Zebrafish NEDD1 (zNEDD1) depletion causes a loss of $\gamma$-tubulin from centrosomes. Cryosections of control or zNEDD1 MO1 + 2-injected embryos were stained for ZNEDD1 and $\gamma$-tubulin at 24 hours post fertilisation (hpf). The region within the marked box is enlarged in the right panels. (a) In the hindbrain of control-injected embryos, zNEDD1 and $\gamma$-tubulin display a high level of expression colocalising in centrosomes of polarised neural cells. (b) Embryos injected with NEDD1 MO1 +2 show reduced centrosomal expression of both zNEDD1 and $\gamma$-tubulin in cells in a similar region of the hindbrain. (c) In control morpholino (MO)-injected embryos in the forebrain there is high expression of zNEDD1 and $\gamma$-tubulin in the centrosomes of highly proliferating cells in the ventricular region. (d) In zNEDD1 MO1 + 2-injected embryos, centrosomal zNEDD1 and $\gamma$-tubulin are both reduced in the forebrain (f). The structures of the ventricles are also disturbed in these embryos. Scale bars $=10 \mu \mathrm{m}$

Figure 6 Zebrafish (NEDD1) zNEDD1 depletion causes disorganised brains and defects in neuronal patterning. (a-l) Uninjected or morpholino (MO)-injected fish were assessed for post-mitotic neurons by HuC antibody staining at 24 hours post fertilisation (hpf). Scale bars $=100 \mu \mathrm{m}$. The eye (e), the forebrain (fb), the midbrain (mb) and the hindbrain $(\mathrm{hb})$. ( $\mathbf{m}-\mathbf{p})$ Uninjected or MO-injected animals were assessed by acetylated $\alpha$-tubulin staining at $24 \mathrm{hpf}$. Embryos are shown from a lateral view. Rohon-Beard cells (RB). Scale bars $=40 \mu \mathrm{m}$. (a-d) Embryos are photographed at $\times 4$ magnification (lateral orientation). (a and b) Uninjected and control-injected embryos show faint but normal HuC staining in different areas of the brain and in the neural tube (arrows). (c) zNEDD1 MO1 + 2-injected embryos are positive for HuC staining, however, they appear morphologically abnormal. (d) This is rescued by co-injection with zNEDD1 mRNA. (e-h) Enlarged lateral images of the neural tube in the trunk show that HuC post-mitotic neurons in this region are largely unaffected by zNEDD1 MO1 +2 injection. (i-I) Enlarged dorsal images of the brain region of embryos show HuC in the forebrain, the hindbrain and the cranial ganglia regions. (i, j) HuC staining is well organised, and the forebrain, the midbrain and the hindbrain regions are clearly defined in uninjected and

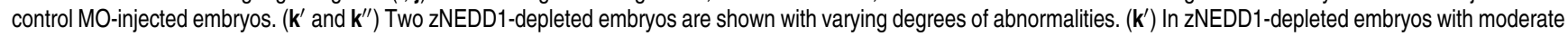
abnormalities, the cranial ganglia are still present but show distorted patterning, and the overall size and definition of the brain structures are reduced. ( $\mathbf{k}^{\prime \prime}$ ) In zNEDD1-depleted embryos with severe abnormalities, the cranial ganglia are more distorted, and all brain structures markedly disorganised and virtually indistinguishable. (I) Normal HuC staining and brain structures after co-injection with zNEDD1 mRNA. ( $m$ and $\mathbf{n}$ ) Uninjected and control-injected embryos show a normal neural tube, with characteristic staining of acetylated $\alpha$-tubulin in RB sensory neurons and their axons within the dorsal longitudinal fasciculus. (o') zNEDD1 MO1 + 2-injected embryos that show mild or moderate abnormalities have a reduced amount of RB cells and fasciculated axons, but the neural tube appears relatively normal. ( $\left.\mathbf{0}^{\prime \prime}\right)$ zNEDD1 MO1 +2 -injected embryos that show severe abnormalities with very few RB cells, a marked reduction in bundling of fasciculated axons, and incomplete neural tube closure. (p) These phenotypes are rescued by co-injected with $Z N E D D 1 \mathrm{mRNA}$ 
or both centrosomes. ${ }^{33}$ Hence, it has been hypothesised that SIL may be required for proper centrosome duplication or for microtubule organisation, as its disruption leads to centrosome dissociation from the mitotic spindle. Therefore, given that the phenotypes observed in embryos depleted of zNEDD1 resemble those of SIL depletion, they are likely to be because, at least in part, of its centrosomal function and in organising a correct mitotic spindle.

There have been limited studies assessing the role of the centrosome in zebrafish development. However, it is apparent that depletion of the other zebrafish centrosomal proteins analysed thus far can also cause defects in the nervous system. Indeed, it has been demonstrated that depletion of the centrosomal protein CEP290 (nephrocystin-6) from zebrafish embryos results in developmental defects of the CNS such as smaller eyes and defects of the brain, ${ }^{36}$ thereby highlighting the importance of centrosomal proteins in CNS development. In addition, depletion of CEP290 causes retinal degeneration and kidney abnormalities, because of its function in cilia. ${ }^{36,37}$ Depletion of other centrosomal proteins, Cep70 and Cep131, also results in embryos with a curved tail, shortened body axis and ectopic otoliths, because of shortened cilia. ${ }^{38}$ Although not examined here, given the link between centrosomal proteins and ciliary function, and the localisation of NEDD1 to the base of cilia in mouse embryos, ${ }^{5}$ it is feasible that zNEDD1 depletion also causes ciliary defects in zebrafish.

As discussed previously, mammalian NEDD1 is essential for the recruitment of $\gamma$-tubulin to the centrosome, where it nucleates microtubules and allows correct mitotic progression. Homologues in Drosophila and Xenopus are not required for this centrosomal $\gamma$-tubulin recruitment. ${ }^{12,13,28}$ It is interesting to note that this study has revealed that the depletion of zNEDD1 from zebrafish also causes a reduction in $\gamma$-tubulin at the centrosome. Hence, zNEDD1 functions to recruit $\gamma$-tubulin to the centrosome in zebrafish, and this is presumably important for correct mitotic progression and embryonic development. It is interesting that zebrafish NEDD1 functions more similarly to mammalian NEDD1 than to the Xenopus homologue, XNEDD1. However, it is possible that the apparent difference in recruitment of $\gamma$-tubulin is due more to the experimental systems, than because of true functional differences.

In conclusion, our study clearly indicates that zNEDD1 interacts with $\gamma$-tubulin and localises to the centrosome as in other species. We have shown that ZNEDD1 is critical for the recruitment of $\gamma$-tubulin to the centrosome, as in mammalian cells, and is essential for the proper development of zebrafish.

\section{Materials and Methods}

In silico analysis. Alignments and phylogenetic trees were carried out using Clustal W2 (http://www. ebi.ac.uk/Tools/clustalw2/index.html). ${ }^{39}$ Protein motif searches were conducted with Scansite Motif Scan (http://scansite.mit.edu/ motifscan_seq.phtml). ${ }^{40}$

Plasmids. Total RNA was extracted from 24 hpf wild-type zebrafish embryos using TRIzol reagent (Invitrogen, Carlsbad, CA, USA). cDNA was synthesised from $2 \mu \mathrm{g}$ RNA using the High-Capacity cDNA Reverse Transcription Kit (Applied Biosystems, Foster City, CA, USA). cDNAs were amplified by RT-PCR using Pfu Ultra II Polymerase (Stratagene, La Jolla, CA, USA) to create various zNEDD1 constructs. All constructs and primer sequences are described in Supplementary Materials and Methods. All DNA constructs were verified by sequencing.

Antibody production. To generate a zNEDD1 antibody, the C-terminal 252 amino acids of the protein fused to GST (pGEX-2T-2-zNEDD1) were expressed and purified as described. ${ }^{41}$ Two rabbits were injected and the serum affinity purified against the zNEDD1 antigen as previously described. ${ }^{42}$

Cell lines, transfections, staining and immunoprecipitations. Human embryonic kidney cells (HEK293T) and mouse NIH-3T3 fibroblasts were cultured in DMEM (Invitrogen), supplemented with 10\% foetal bovine serum, $50 \mathrm{U} \mathrm{ml}^{-1}$ penicillin, $0.05 \mathrm{mg} \mathrm{ml}^{-1}$ streptomycin (all from Invitrogen) at $37^{\circ} \mathrm{C}$ in $5 \% \mathrm{CO}_{2}$. Transfections were carried out using Lipofectamine 2000 (Invitrogen), according to the manufacturer's instructions. Cell staining was conducted as described using 1:500 rabbit $\alpha$-zNEDD $1^{5}$ and $1: 500$ mouse $\alpha-\gamma$-tubulin, (GTU-88, Sigma, St. Louis, MO, USA) and images obtained using a confocal microscope as described. ${ }^{43}$ For immunoprecipitation, $1 \times 10^{6}$ HEK293T cells were lysed and immunoprecipitated as previously described, ${ }^{44}$ using $1 \mu \mathrm{l}$ of antibodies to zNEDD1 or $\alpha-\gamma$-tubulin.

SDS-PAGE and immunoblotting. Samples were boiled for $5 \mathrm{~min}$ in $2 \times$ SDS loading buffer $(100 \mathrm{mM}$ Tris- $\mathrm{HCl}$ pH $6.8,200 \mathrm{mM}$ DTT, $4 \%$ SDS, $0.2 \%$ bromophenol blue and $20 \%$ glycerol), separated by SDS-PAGE and transferred to PVDF membranes. Immunoblotting for ZNEDD1 was carried out using rabbit $\alpha$-zNEDD1 at $1: 200$ as described. ${ }^{5}$ Immunoblotting for $\gamma$-tubulin and $\beta$-actin were carried out using mouse $\alpha-\gamma$-tubulin (GTU-88, Sigma) and mouse $\alpha$ - $\beta$-actin (AC- 15 , Sigma). ${ }^{44}$ The intensity of protein bands was quantitated and normalised to $\gamma$-tubulin bands using ImageQuant (Molecular Dynamics, Sunnyvale, CA, USA).

Zebrafish maintenance and staging. Zebrafish were maintained in the Adelaide Zebrafish Facility (Adelaide University, Adelaide, SA, Australia) at $28.5^{\circ} \mathrm{C}$ under standard conditions. ${ }^{45}$ Embryos were collected and maintained in a medium containing $13.72 \mathrm{mM} \mathrm{NaCl}, 0.54 \mathrm{mM} \mathrm{KCl}, 0.025 \mathrm{mM} \mathrm{Na}_{2} \mathrm{HPO}_{4}, 0.044 \mathrm{mM} \mathrm{K}_{2} \mathrm{HPO}_{4}$, $1 \mathrm{mM} \mathrm{CaCl}_{2}, 1 \mathrm{mM} \mathrm{MgSO}_{4}$ and $0.035 \%$ (w/v) $\mathrm{NaHCO}_{3}$. Developmental stages were determined by using both hours post fertilisation and morphological features. ${ }^{9}$ Before manipulation, embryos were anesthetised in $200 \mu \mathrm{g} \mathrm{ml}^{-1}$ tricaine (Sigma) and where necessary, dechorionated manually.

In situ hybridisation. Anti-sense and sense digoxygen (DIG) labelled riboprobes were generated from a pGEM-T-Easy plasmid containing amino acids 1-422 of ZNEDD1. The plasmid was linearised at the $5^{\prime}$ or $3^{\prime}$ end of the clone using Hincll or Notl enzymes, respectively. RNA was transcribed in vitro, incorporating DIG-11-UTP by T7 or T3 RNA polymerase for the sense and anti-sense probes respectively, using the DIG RNA labelling kit (Roche, Mannheim, Germany). Hybridisations were conducted as described ${ }^{46}$ with the following modifications: for embryos older than $24 \mathrm{hpf}$, embryo medium was replaced at $10 \mathrm{hpf}$ with $0.0045 \%$ phenylthiourea (Sigma) in embryo medium to prevent pigmentation. Chorions were removed manually with forceps after fixation. After incubating with $\alpha$-DIG (1:5000, Roche) and staining solution, embryos were submerged in $2: 1$ benzyl benzoate/ benzyl alcohol mix (Sigma). Embryos were then rehydrated by 5 min washes of 75 , 50 and $25 \%$ methanol in PBST, washed in PBST and cleared in $80 \%$ glycerol/PBST (PBS/0.1\% Tween20) overnight before being mounted. A Zeiss Axiophot microscope was used with DIC (Nomarski differential interference contrast) optics. Images were captured using a $\mathrm{HC} 1000$ digital $3 \mathrm{CCD}$ colour camera (Fuji, Dusseldorf, Germany) and compiled using Adobe Photoshop 6.0 software.

Protein extraction from zebrafish. To extract protein from zebrafish, embryos were dechorionated and the yolks were removed manually with forceps and needles. The embryos were then vortexed in Ringers solution $(55 \mathrm{mM} \mathrm{NaCl}$, $1.8 \mathrm{mM} \mathrm{KCl}$ and $1.25 \mathrm{mM} \mathrm{NaHCO}_{3}$ ) for $5 \mathrm{~min}$ to dissolve the remaining yolk, before pelleting by centrifugation at $4000 \times g$ for $5 \mathrm{~min}$ and lysis in RIPA buffer (50 mM Tris-HCl, pH 7.5, $150 \mathrm{mM} \mathrm{NaCl}, 1 \%$ NP40, 0.5\% deoxycholic acid, $0.1 \%$ SDS, $10 \mathrm{mM}$ EDTA and complete protease inhibitor cocktail (Roche)). Protein concentration was assessed using the BioRad Protein Assay (Biorad Laboratories, Hercules, CA, USA).

MO oligo design and injection. Morpholino oligos were designed and synthesised by GeneTools (Philomath, OR, USA) and resuspended in nuclease free 
$\mathrm{MQ} \mathrm{H}_{2} \mathrm{O}$ to give a stock solution of $2 \mathrm{mM}$. Anti-sense $\mathrm{MO}$ sequences are given in the $5^{\prime} \rightarrow 3^{\prime}$ direction.

ZNEDD1 M01: ACGTCCTCCATCCCAGCAGCCTTGT

ZNEDD1 MO2: AAGTGTAGACAATGTAAATGATATG

Control MO: AAGTGTAGACAATGTAAATGATATG

p53 MO: GCGCCATTGCTTTGCAAGAATTG

Before microinjection, $\mathrm{MO}$ stock was diluted to the required concentration in $\mathrm{MQ}$ $\mathrm{H}_{2} \mathrm{O}$. For all experiments involving microinjection, MOs were injected into the cytoplasm of the one-cell stage embryos using an MPPI-2 Pressure Injector (Applied Scientific Instrumentation, Eugene, OR, USA). Injected embryos were maintained at $28.5^{\circ} \mathrm{C}$ in embryo media.

Generation of capped mRNA for rescue and overexpression. For rescue experiments, zNEDD1 in pBlueScript KS was linearised with Sacll and used to generate capped mRNA with the $\mathrm{G}\left(5^{\prime}\right) \mathrm{ppp}\left(5^{\prime}\right) \mathrm{G}$ cap analogue (Ambion, Austin TX, USA) using the MEGAscript T7 kit (Ambion), according to the manufacturer's instructions, except that a cap analog/GTP ratio of $4: 1$ was used. For GFP overexpression, zNEDD1 598-676 in pcDNA-DEST53 or pcDNA-DEST53 alone was linearised with $\mathrm{Pacl}$ or $E c \mathrm{RI}$ respectively and capped mRNA generated as described above.

Light and epifluorescence microscopy. The embryos were anaesthetised $24 \mathrm{~h}$ after injection, mounted onto a glass slide and photographed at $\times 4$ and $\times 10$ on a BX51 microscope (Olympus, Center Valley, PA, USA) with UPlanApo objectives with a 488 and $568 \mathrm{~nm}$ filter fitted with a DP70 camera (Olympus) and processed with Olysia Bioreport software (Olympus).

Detection of apoptosis by TUNEL in whole-mount embryos. Apoptosis was detected by TUNEL using the In situ Cell Death Detection Kit, TMR Red (Roche). ${ }^{47}$ Images were acquired on a BX51 microscope as described above.

Whole-mount immunohistochemistry for $\mathrm{pH} 3$ and acetylated $\boldsymbol{\alpha}$-tubulin. Embryos were fixed and permeabilised in $100 \%$ methanol overnight at $-20^{\circ} \mathrm{C}$ and rehydrated by 5 min washes of $75,50,25 \%$ methanol in PBST, before $2 \times 10$ min washes in PBST. Embryos were then incubated in blocking solution $(0.1 \%$ BSA, $10 \%$ goat serum in PBST) for at least $1 \mathrm{~h}$ prior to incubation with the primary antibodies $(1: 100$ rabbit $\alpha$-phosphorylated histone $\mathrm{H} 3, \mathrm{C} 8$, Cell Signalling, Beverly, MA, USA; $1: 500$ mouse acetylated $\alpha$-tubulin, 6-11B-1, Sigma). Following incubation overnight at $4^{\circ} \mathrm{C}$ with gentle rocking and washing with PBST, embryos were incubated with secondary antibody (1:1000 rabbit or mouse Alexa Fluor 488, Molecular Probes, Invitrogen) for at least $2 \mathrm{~h}$ at RT with gentle rocking. Embryos were then washed in PBST for $3 \mathrm{~h}$, cleared overnight in PBST $/ 80 \%$ glycerol and mounted. Embryos stained with $\alpha-\mathrm{pH} 3$ were photographed as for TUNEL staining. Embryos stained with $\alpha$-acetylated tubulin were photographed on a confocal microscope as described. ${ }^{43}$

Whole-mount immunohistochemistry for HuC. Embryos were fixed in $4 \% \mathrm{PFA} / \mathrm{PBS}$ overnight followed by several washes in PBST. Embryos were then incubated on a rotor with $0.5 \% \mathrm{H}_{2} \mathrm{O}_{2}$ and $0.5 \%$ goat serum for $30 \mathrm{~min}$ at RT, washed in PBST for $10 \mathrm{~min}$, and then blocked and stained with 1:1000 rabbit $\alpha$-HuC (16A11, Molecular Probes, Invitrogen) using the Elite mouse IgG Vectastain ABC Kit (Vector Laboratories, Burlingame, CA, USA). For development of colour the DAB substrate kit for peroxidise was used (Vector Laboratories). Embryos were then washed in PBST, cleared, mounted and photographed as for light microscopy.

OCT sectioning and immunohistochemistry. For sectioning, embryos were fixed in $100 \%$ methanol overnight at $-20^{\circ} \mathrm{C}$, rehydrated in 5 min washes of 75 50 and $25 \%$ methanol in PBST, before $2 \times 10$ min washes in PBST. Embryos were submerged in $30 \%$ sucrose/PBST for at least $3 \mathrm{~h}$, before embedding into TissueTek OCT compound (Sakura Finetek, Torrance, CA, USA), frozen on dry ice and sectioned at $10 \mu \mathrm{m}$ onto glass slides using a cryostat (CM1950, Leica, Wetzlar, Germany). Sections were fixed and stained as described using 1:200 rabbit $\alpha$-ZNEDD $1^{5}$ and $1: 500$ mouse $\alpha-\gamma$-tubulin (GTU-88, Sigma).

\section{Conflict of interest}

The authors declare no conflict of interest.
Acknowledgements. This work was supported in part by an Australian Postgraduate Award to JAM and a NHMRC Senior Principal Research Fellowship to SK.

1. Kumar S, Matsuzaki T, Yoshida Y, Noda M. Molecular cloning and biological activity of a novel developmentally regulated gene encoding a protein with beta-transducin-like structure. J Biol Chem 1994; 269: 11318-11326.

2. Haren L, Remy MH, Bazin I, Callebaut I, Wright M, Merdes A. NEDD1-dependent recruitment of the gamma-tubulin ring complex to the centrosome is necessary for centriole duplication and spindle assembly. J Cell Biol 2006; 172: 505-515.

3. Luders J, Patel UK, Stearns T. GCP-WD is a gamma-tubulin targeting factor required for centrosomal and chromatin-mediated microtubule nucleation. Nat Cell Biol 2006; 8: 137-147.

4. Tillement V, Haren L, Roullet N, Etievant C, Merdes A. The centrosome protein NEDD1 as a potential pharmacological target to induce cell cycle arrest. Mol Cancer 2009; 8: 10.

5. Manning JA, Colussi PA, Koblar SA, Kumar S. Nedd1 expression as a marker of dynamic centrosomal localization during mouse embryonic development. Histochem Cell Biol 2008; 129: $751-764$.

6. Hudson JW, Kozarova A, Cheung P, Macmillan JC, Swallow CJ, Cross JC et al. Late mitotic failure in mice lacking Sak, a polo-like kinase. Curr Biol 2001; 11: 441-446.

7. Yuba-Kubo A, Kubo A, Hata M, Tsukita S. Gene knockout analysis of two gamma-tubulin isoforms in mice. Dev Biol 2005; 282: 361-373.

8. Shepard JL, Stern HM, Pfaff KL, Amatruda JF. Analysis of the cell cycle in zebrafish embryos. Methods Cell Biol 2004; 76: 109-125.

9. Kimmel CB, Ballard WW, Kimmel SR, Ullmann B, Schilling TF. Stages of embryonic development of the zebrafish. Dev Dyn 1995; 203: 253-310.

10. Eisen JS, Smith JC. Controlling morpholino experiments: don't stop making antisense. Development 2008; 135: 1735-1743.

11. Kane DA. Cell cycles and development in the embryonic zebrafish. Methods Cell Biol 1999; 59: $11-26$

12. Gunawardane RN, Martin OC, Zheng Y. Characterization of a new gammaTuRC subunit with WD repeats. Mol Biol Cell 2003; 14: 1017-1026.

13. Liu L, Wiese C. Xenopus NEDD1 is required for microtubule organization in Xenopus egg extracts. J Cell Sci 2008; 121: 578-589.

14. Zeng CJ, Lee YR, Liu B. The WD40 repeat protein NEDD1 functions in microtubule organization during cell division in Arabidopsis thaliana. Plant Cell 2009; 21: 1129-1140.

15. Smith TF, Gaitatzes $C$, Saxena K, Neer EJ. The WD repeat: a common architecture for diverse functions. Trends Biochem Sci 1999; 24: 181-185.

16. Wullimann MF, Knipp S. Proliferation pattern changes in the zebrafish brain from embryonic through early postembryonic stages. Anat Embryol (Berl) 2000; 202: 385-400.

17. Pelegri F. Maternal factors in zebrafish development. Dev Dyn 2003; 228: 535-554.

18. Kane DA, Kimmel CB. The zebrafish midblastula transition. Development 1993; 119: 447-456.

19. Schenck A, Goto-Silva L, Collinet C, Rhinn M, Giner A, Habermann B et al. The endosomal protein Appl1 mediates Akt substrate specificity and cell survival in vertebrate development. Cell 2008; 133: 486-497.

20. Robu ME, Larson JD, Nasevicius A, Beiraghi S, Brenner C, Farber SA et al. p53 activation by knockdown technologies. PLOS Genet 2007; 3: e78.

21. Nowak M, Koster $\mathrm{C}$, Hammerschmidt M. Perp is required for tissue-specific cell survival during zebrafish development. Cell Death Differ 2005; 12: 52-64.

22. Hendzel MJ, Wei $Y$, Mancini MA, Van Hooser A, Ranalli T, Brinkley BR et al. Mitosis-specific phosphorylation of histone $\mathrm{H} 3$ initiates primarily within pericentromeric heterochromatin during $\mathrm{G} 2$ and spreads in an ordered fashion coincident with mitotic chromosome condensation. Chromosoma 1997; 106: 348-360.

23. Kim CH, Ueshima E, Muraoka O, Tanaka H, Yeo SY, Huh TL et al. Zebrafish elav/HuC homologue as a very early neuronal marker. Neurosci Lett 1996; 216: 109-112.

24. Mawdsley DJ, Cooper HM, Hogan BM, Cody SH, Lieschke GJ, Heath JK. The Netrin receptor Neogenin is required for neural tube formation and somitogenesis in zebrafish. Dev Biol 2004; 269: 302-315.

25. Park HC, Hong SK, Kim HS, Kim SH, Yoon EJ, Kim CH et al. Structural comparison of zebrafish Elav/Hu and their differential expressions during neurogenesis. Neurosci Lett 2000; 279: 81-84.

26. Cambray-Deakin MA, Burgoyne RD. Posttranslational modifications of alpha-tubulin: acetylated and detyrosinated forms in axons of rat cerebellum. $J$ Cell Biol 1987; 104: 1569-1574.

27. Bernhardt RR, Chitnis $A B$, Lindamer L, Kuwada JY. Identification of spinal neurons in the embryonic and larval zebrafish. J Comp Neurol 1990; 302: 603-616.

28. Verollet C, Colombie N, Daubon T, Bourbon HM, Wright M, Raynaud-Messina B. Drosophila melanogaster gamma-TuRC is dispensable for targeting gamma-tubulin to the centrosome and microtubule nucleation. J Cell Biol 2006; 172: 517-528.

29. Kimmel CB. Patterning the brain of the zebrafish embryo. Annu Rev Neurosci 1993; 16 : 707-732.

30. Higginbotham HR, Gleeson JG. The centrosome in neuronal development. Trends Neurosci 2007; 30: 276-283.

31. Meulmeester E, Jochemsen AG. p53: a guide to apoptosis. Curr Cancer Drug Targets 2008; 8: 87-97. 
32. Lowery LA, Sive $\mathrm{H}$. Initial formation of zebrafish brain ventricles occurs independently of circulation and requires the nagie oko and snakehead/atp1a1a.1 gene products. Development 2005; 132: 2057-2067.

33. Pfaff KL, Straub CT, Chiang K, Bear DM, Zhou Y, Zon LI. The zebra fish cassiopeia mutant reveals that SIL is required for mitotic spindle organization. Mol Cell Biol 2007; 27: 5887-5897.

34. Aplan PD, Lombardi DP, Ginsberg AM, Cossman J, Bertness VL, Kirsch IR. Disruption of the human SCL locus by 'illegitimate' V-(D)-J recombinase activity. Science 1990; 250: 1426-1429.

35. Izraeli S, Colaizzo-Anas T, Bertness VL, Mani K, Aplan PD, Kirsch IR. Expression of the $\mathrm{SIL}$ gene is correlated with growth induction and cellular proliferation. Cell Growth Differ 1997; 8: 1171-1179.

36. Sayer JA, Otto EA, O'Toole JF, Nurnberg G, Kennedy MA, Becker $C$ et al. The centrosomal protein nephrocystin- 6 is mutated in Joubert syndrome and activates transcription factor ATF4. Nat Genet 2006; 38: 674-681.

37. Chang B, Khanna H, Hawes N, Jimeno D, He S, Lillo $C$ et al. In-frame deletion in a novel centrosomal/ciliary protein CEP290/NPHP6 perturbs its interaction with RPGR and results in early-onset retinal degeneration in the rd16 mouse. Hum Mol Genet 2006; 15: 1847-1857.

38. Wilkinson CJ, Carl M, Harris WA. Cep70 and Cep131 contribute to ciliogenesis in zebrafish embryos. BMC Cell Biol 2009; 10: 17.

39. Larkin MA, Blackshields G, Brown NP, Chenna R, McGettigan PA, McWilliam $\mathrm{H}$ et al. Clustal W and Clustal X version 2.0. Bioinformatics 2007; 23: 2947-2948.
40. Obenauer JC, Cantley LC, Yaffe MB. Scansite 2.0: proteome-wide prediction of cell signaling interactions using short sequence motifs. Nucleic Acids Res 2003; 31: 3635-3641.

41. Harvey KF, Dinudom A, Komwatana P, Jolliffe CN, Day ML, Parasivam G et al. All three WW domains of murine Nedd4 are involved in the regulation of epithelial sodium channels by intracellular $\mathrm{Na}+$. J Biol Chem 1999; 274: 12525-12530.

42. Konstas AA, Shearwin-Whyatt LM, Fotia AB, Degger B, Riccardi D, Cook DI et al. Regulation of the epithelial sodium channel by N4WBP5A, a novel Nedd4/Nedd4-2interacting protein. J Biol Chem 2002; 277: 29406-29416.

43. Foot NJ, Dalton HE, Shearwin-Whyatt LM, Dorstyn L, Tan SS, Yang B et al. Regulation of the divalent metal ion transporter DMT1 and iron homeostasis by a ubiquitin-dependent mechanism involving Ndfips and WWP2. Blood 2008; 112: 4268-4275.

44. Shearwin-Whyatt LM, Brown DL, Wylie FG, Stow JL, Kumar S. N4WBP5A (Ndfip2), a Nedd4-interacting protein, localizes to multivesicular bodies and the Golgi, and has a potential role in protein trafficking. J Cell Sci 2004; 117: 3679-3689.

45. Westerfield M. The Zebrafish Book: A Guide to the Laboratory Use of Zebrafish (Danio Rerio), 3rd edn. University of Oregon Press: Eugene, 1995

46. Thisse $\mathrm{C}$, Thisse B. High-resolution in situ hybridization to whole-mount zebrafish embryos. Nat Protoc 2008; 3: 59-69.

47. Berghmans S, Murphey RD, Wienholds E, Neuberg D, Kutok JL, Fletcher CD et al. tp53 mutant zebrafish develop malignant peripheral nerve sheath tumors. Proc Natl Acad Sci USA 2005; 102: 407-412.

Supplementary Information accompanies the paper on Cell Death and Differentiation website (http://www.nature.com/cdd) 